\title{
Searches for new physics with heavy flavour at ATLAS
}

\author{
Semen Turchikhin* \\ On behalf of the ATLAS Collaboration \\ ${ }^{1}$ Joint Institute for Nuclear Research
}

\begin{abstract}
The Flavour Changing Neutral Current processes are sensitive to New Physics contributions, in particular through additional electroweak loop amplitudes. The angular analysis of the decay of $B^{0} \rightarrow \mu^{+} \mu^{-} K^{* 0}$ is presented. A number of angular coefficients are measured as a function of the invariant mass squared of the dimuon system using data collected by the ATLAS experiment at the LHC. Comparison is made to theoretical predictions, including for the observable $P_{5}^{\prime}$, for which there has been recent tension between theory and experiment. ATLAS result on the study of the $B_{(s)}^{0} \rightarrow \mu^{+} \mu^{-}$rare decays is also presented.
\end{abstract}

\section{Introduction}

Studying heavy flavour decay processes at collider experiments provides an excellent opportunity for searches of indirect manifestations of new physics. Rare decays of $b$ hadrons involving $b \rightarrow$ sl $\ell$ transition belong to such kind of processes. They are mediated by flavour-changing neutral currents which are forbidden at tree level within the Standard Model (SM) and occur at higher orders via loop diagrams. Thus these processes are sensitive to contributions of heavy particles predicted by various models beyond the SM in the loops.

This proceeding presents two analyses of such decays recently performed in the ATLAS experiment [1] at Large Hadron Collider: measurement of angular distributions in the $B^{0} \rightarrow \mu^{+} \mu^{-} K^{* 0}$ semileptonic decay [2] and study of $B_{(s)}^{0} \rightarrow \mu^{+} \mu^{-}$rare decay [3].

\section{Angular analysis of $B^{0} \rightarrow \mu^{+} \mu^{-} K^{* 0}$ decay}

Decay $B^{0} \rightarrow \mu^{+} \mu^{-} K^{* 0}$ occurs via loop diagrams and has a branching ratio of $(1.03 \pm 0.06) \times 10^{-6}$ [4]. Angular distributions of the decay products are observables of interest for such process as they are potentially sensitive to different types of new physics.

Analysis of the $B^{0} \rightarrow \mu^{+} \mu^{-} K^{* 0}$ decay is performed using the $p p$ collisions data sample collected by the ATLAS detector at centre of mass energy $\sqrt{s}=8 \mathrm{TeV}$ corresponding to an integrated luminosity of $20.3 \mathrm{fb}^{-1}$ [2].

The kinematics of the $B^{0} \rightarrow \mu^{+} \mu^{-} K^{* 0}$ decay $^{1}$ (with subsequent $K^{* 0} \rightarrow K^{+} \pi^{-}$) is described in terms of four variables: the dimuon invariant mass squared, $q^{2}$, and three angles describing the final state geometrical configuration, as illustrated in Fig. 1.

\footnotetext{
^e-mail: Semen.Turchikhin@cern.ch

${ }^{1}$ Charge conjugation is implied here and below unless stated otherwise.
} 


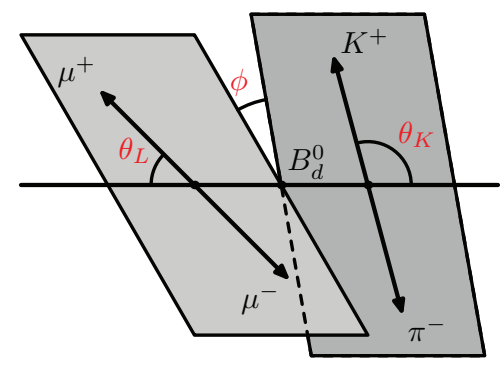

Figure 1. Illustration of the kinematic angles $\left(\phi, \theta_{L}, \theta_{K}\right)$ definition in $B^{0} \rightarrow \mu^{+} \mu^{-} K^{* 0}\left(K^{+} \pi^{-}\right)$decay.

The differential decay rate as a function of the decay angles can be expressed as

$$
\begin{aligned}
\frac{1}{\mathrm{~d} \Gamma / \mathrm{d} q^{2}} \frac{\mathrm{d}^{4} \Gamma}{\mathrm{d} \cos \theta_{L} \mathrm{~d} \cos \theta_{K} \mathrm{~d} \phi \mathrm{d} q^{2}}= & \frac{9}{32 \pi}\left[\frac{3\left(1-F_{L}\right)}{4} \sin ^{2} \theta_{L}+F_{L} \cos ^{2} \theta_{K}+\frac{1-F_{L}}{4} \sin ^{2} \theta_{K} \cos 2 \theta_{L}\right. \\
& -F_{L} \cos ^{2} \theta_{K} \cos 2 \theta_{L}+S_{3} \sin ^{2} \theta_{K} \sin ^{2} \theta_{L} \cos 2 \phi \\
& +S_{4} \sin 2 \theta_{K} \sin 2 \theta_{L} \cos \phi+S_{5} \sin 2 \theta_{K} \sin \theta_{L} \cos \phi \\
& +S_{6} \sin ^{2} \theta_{K} \cos \theta_{L}+S_{7} \sin 2 \theta_{K} \sin \theta_{L} \sin \phi \\
& \left.+S_{8} \sin 2 \theta_{K} \sin 2 \theta_{L} \sin \phi+S_{9} \sin ^{2} \theta_{K} \sin ^{2} \theta_{L} \sin 2 \phi\right]
\end{aligned}
$$

where $F_{L}$ is the fraction of longitudinally polarised $K^{* 0}$ 's and $S_{i}$ are the angular coefficients related to the decay transversity amplitudes. Extracting the full set of these parameters from a fit to data using the above equation requires sufficient statistics and purity of the data sample which are not available with the analysed dataset. To mitigate this, trigonometric transformations are used to simplify the fit so that terms in Eq. (1) drop out. The folding schema used in the analysis follows Ref. [5] and results in four sets of transformations, such that three parameters can be extracted from each of four fits: $F_{L}$, $S_{3}$ and one of the other $S_{i}$ parameters. As a consequence, $S_{6}$ and $S_{9}$ cannot be extracted from the data.

The $S_{i}$ parameters are sensitive to hadronic form factors, and to reduce the corresponding theoretical uncertainties, the following optimized set of parameters is used in the analysis:

$$
P_{1}=\frac{2 S_{3}}{1-F_{L}}, \quad P_{2}=\frac{1}{2} \frac{S_{6}}{1-F_{L}}, \quad P_{3}=-\frac{S_{9}}{1-F_{L}}, \quad P_{j=4,5,6,8}^{\prime}=\frac{S_{i=4,5,7,8}}{\sqrt{F_{L}\left(1-F_{L}\right)}} .
$$

The $B^{0}$ decay candidates are reconstructed by fitting a common vertex of two oppositely charged muons and two other oppositely charged tracks forming a $K^{* 0}$ meson. Signal candidate selection mostly focuses on combinatorial background suppression. Selection criteria include a number of cuts on kinematic properties, quality of the vertex fit, displacement of the decay vertex and pointing of the candidate momentum towards the primary interaction point. Further signal fits are performed for the candidates with the $q^{2}$ value within the range $[0.04,6] \mathrm{GeV}^{2}$ excluding $[0.98,1.1] \mathrm{GeV}^{2}$ due to background from $\phi \rightarrow \mu^{+} \mu^{-}$decays. In this $q^{2}$ range, 787 signal candidates are retained after the full selection.

Extended unbinned maximum likelihood fits of the distributions of $B^{0}$ candidate mass and angular variables $\cos \theta_{K}, \cos \theta_{L}$, and $\phi$ are performed on the data to extract the angular parameters. The fit model and procedure have been extensively validated using simulated pseudo-experiments. The fits 


\begin{tabular}{r|cc}
\hline \hline$q^{2}\left[\mathrm{GeV}^{2}\right]$ & $n_{\text {signal }}$ & $n_{\text {background }}$ \\
\hline$[0.04,2.0]$ & $128 \pm 22$ & $122 \pm 22$ \\
{$[2.0,4.0]$} & $106 \pm 23$ & $113 \pm 23$ \\
{$[4.0,6.0]$} & $114 \pm 24$ & $204 \pm 26$ \\
\hline$[0.04,4.0]$ & $236 \pm 31$ & $233 \pm 32$ \\
{$[1.1,6.0]$} & $275 \pm 35$ & $363 \pm 36$ \\
{$[0.04,6.0]$} & $342 \pm 39$ & $445 \pm 40$ \\
\hline \hline
\end{tabular}

Table 1. The values of fitted signal, $n_{\text {signal }}$, and background, $n_{\text {background }}$, yields obtained for different regions of $q^{2}$. The uncertainties indicated are statistical. Table taken from Ref. [2].

are done separately in three non-overlapping regions of $q^{2}:[0.04,2.0],[2.0,4.0]$, and $[4.0,6.0] \mathrm{GeV}^{2}$, as well as in wider bins $[0.04,4.0],[1.1,6.0],[0.04,6.0] \mathrm{GeV}^{2}$, to facilitate comparison with the results of other experiments and theoretical predictions. The signal and background yields in these $q^{2}$ bins are shown in Table 1.

A number of sources of systematic uncertainty affecting the analysis are studied. The methods for determining these uncertainties are based on either a comparison of nominal and modified fit results, or on observed fit biases in modified pseudo-experiments. Examples of the most significant sources are: various background contributions producing peaks in the distributions of the angular variables; uncertainty of background angular shapes modelling; detector angular acceptance description; detector alignment and magnetic field calibration uncertainties; effect of intrinsic bias of the maximum likelihood estimator.

The distributions of $F_{L}$ and $P_{i}^{(\prime)}$ parameters as a function of $q^{2}$ are shown in Fig. 2. The figure also shows results of theoretical predictions of SM by various groups: Ciuchini et al. (CFFMPSV) [6], Descotes-Genon et al. (DHMV) [7], and Jäger and Martin Camalich (JC) [8]. Results of measurements from LHCb [9], CMS [10], Belle [11, 12] and BaBar [13] experiments are also shown.

Theoretical predictions are in a good agreement with the ATLAS result, except for the $P_{4}^{\prime}$ and $P_{5}^{\prime}$ measurements in $q^{2} \in[4.0,6.0] \mathrm{GeV}^{2}$ and $P_{8}^{\prime}$ in $q^{2} \in[2.0,4.0] \mathrm{GeV}^{2}$. The observed deviations of $P_{4}^{\prime}$ and $P_{5}^{\prime}$ from the SM expectations are consistent with those reported by LHCb [9] and are 2.5 and 2.7 standard deviations from the DHMV predictions, respectively (with less significant difference for the other calculations). All measurements are found to be within three standard deviations of the range covered by the different predictions.

\section{Study of $B_{(s)}^{0} \rightarrow \mu^{+} \mu^{-}$rare decay}

Decays of $B_{s}^{0}$ or $B^{0}$ mesons into a muon pair represent another example of $b \rightarrow$ sll transition. Measurement of their branching fractions is of particular interest because of their additional helicity suppression and since they can be predicted by the SM very precisely: $\mathcal{B}\left(B_{s}^{0} \rightarrow \mu^{+} \mu^{-}\right)=$ $(3.65 \pm 0.23) \times 10^{-9}$ and $\mathcal{B}\left(B^{0} \rightarrow \mu^{+} \mu^{-}\right)=(1.06 \pm 0.09) \times 10^{-10}$ [14]. Being a clear experimental signature, these decays are considered to be one of the most prominent probes for new physics in heavy flavour sector. The CMS and LHCb collaborations so far have reported the observation of $B_{s}^{0} \rightarrow \mu^{+} \mu^{-}[15,16]$ and evidence of $B^{0} \rightarrow \mu^{+} \mu^{-}$decays, with combined values: $\mathcal{B}\left(B_{s}^{0} \rightarrow \mu^{+} \mu^{-}\right)=\left(2.8_{-0.6}^{+0.7}\right) \times 10^{-9}$ and $\mathcal{B}\left(B^{0} \rightarrow \mu^{+} \mu^{-}\right)=\left(3.9_{-1.4}^{+1.6}\right) \times 10^{-10}[17]$.

ATLAS analysis of the $B_{(s)}^{0} \rightarrow \mu^{+} \mu^{-}$decays presented below uses the full Run-1 pp collision dataset collected at centre of mass energies $\sqrt{s}=7$ and $8 \mathrm{TeV}$ and corresponding to an integrated luminosity of $25 \mathrm{fb}^{-1}$ [3]. 

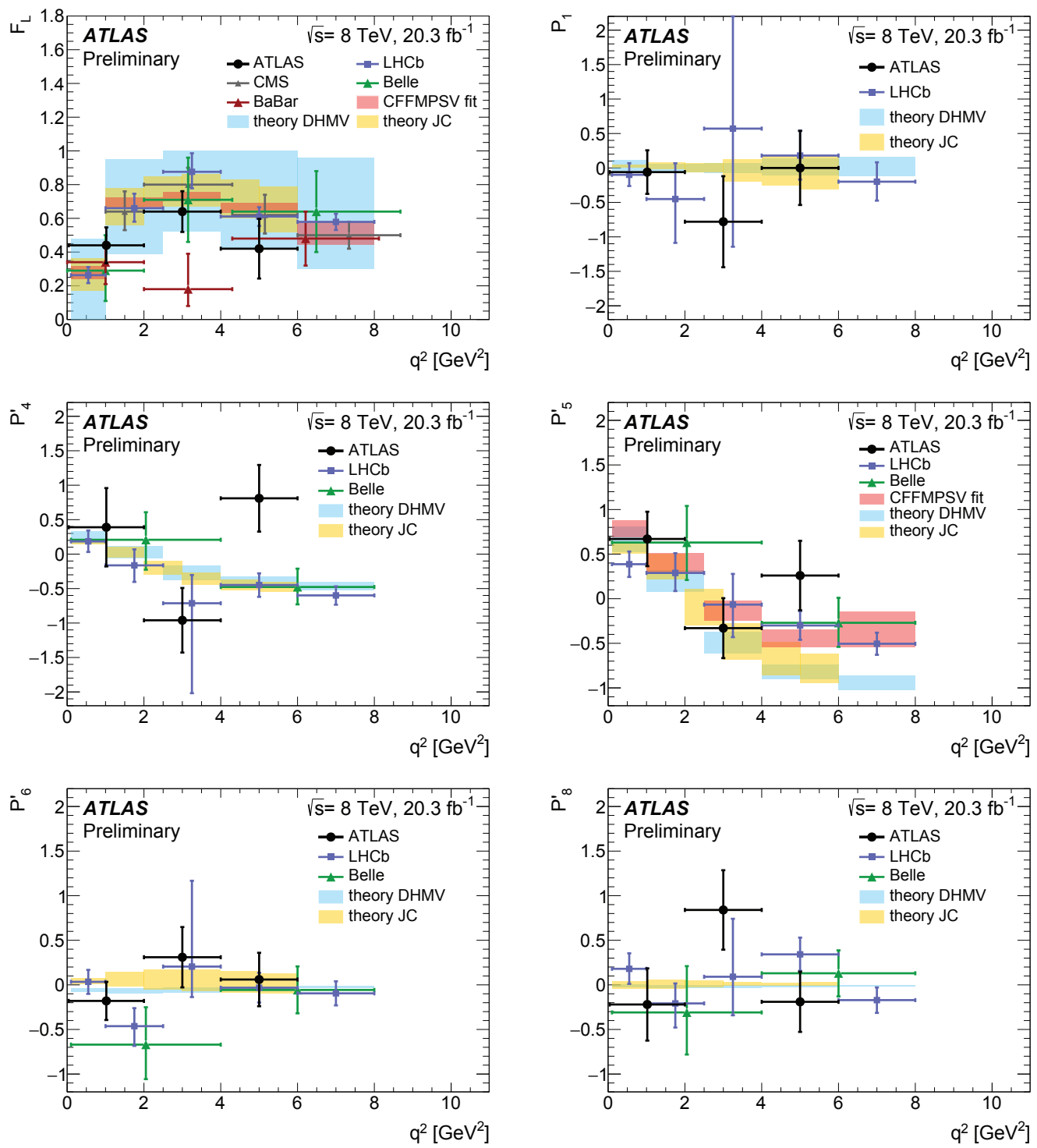

Figure 2. The measured values of $F_{L}$ and $P_{i}^{(\prime)}$ compared with predictions from the theoretical groups and other experiment results. Figure taken from Ref. [2].

The branching fractions of the signal decay modes are measured relative to the normalisation decay $B^{+} \rightarrow J / \psi\left(\mu^{+} \mu^{-}\right) K^{+}$that has a known branching fraction [4]. This approach allows to substantially reduce systematic uncertainties related to the luminosity, detector acceptance, reconstruction and trigger efficiencies. The signal decay branching fraction is thus given by the following equation:

$$
\mathcal{B}\left(B_{(s)}^{0} \rightarrow \mu^{+} \mu^{-}\right)=\frac{N_{d(s)}}{\varepsilon_{\mu^{+} \mu^{-}}} \times\left[\mathcal{B}\left(B^{+} \rightarrow J / \psi K^{+}\right) \times \mathcal{B}\left(J / \psi \rightarrow \mu^{+} \mu^{-}\right)\right] \frac{\varepsilon_{J / \psi K^{+}}}{N_{J / \psi K^{+}}} \times \frac{f_{u}}{f_{d(s)}},
$$


where $N_{d}\left(N_{s}\right)$ is the $B^{0} \rightarrow \mu^{+} \mu^{-}\left(B_{s}^{0} \rightarrow \mu^{+} \mu^{-}\right)$signal yield, $N_{J / \psi K^{+}}$is the normalisation channel yield, $\varepsilon_{\mu^{+} \mu^{-}}$and $\varepsilon_{J / \psi K^{+}}$are the corresponding values of acceptance times efficiency evaluated from simulation, and $f_{u} / f_{d}\left(f_{u} / f_{s}\right)$ is the known ratio of the hadronisation probabilities of a $b$-quark into $B^{+}$and $B^{0}\left(B_{s}^{0}\right)$. The yield of the normalisation channel is obtained from a maximum likelihood fit of $J / \psi K^{+}$invariant mass distribution in data.

A blind analysis is performed which means that data in the dimuon invariant mass region from 5166 to $5526 \mathrm{MeV}$ are removed until the procedures for event selection and the signal yield extraction are completely defined.

Multivariate technique based on boosted decision trees (BDT) [18] is used for event selection of both signal and normalisation decay channels in the data. Two BDT classifiers are used. The first one referred to as "continuum BDT" is intended to separate the signal from a huge combinatorial background of pairs of muons coming from different sources (continuum background). It takes as input a number of variables characterising the decay candidate kinematics, properties of decay vertex, isolation of the decay candidate. The classifier is trained and tested using simulated samples of signal and background. The second classifier is used for suppression of mis-identified muons and exploits properties of their reconstructed tracks. This mis-identification can happen if a charged pion or kaon decays within the detector volume or punches through the calorimeters to the ATLAS muon spectrometer. If two hadrons from $B \rightarrow h h^{\prime}$ ( $h, h^{\prime}$ being charged pions or kaons) decays produce such fake muons, this signature mimics the signal decay thus being a source of irreducible background.

The yields of the decays $B_{s}^{0} \rightarrow \mu^{+} \mu^{-}$and $B^{0} \rightarrow \mu^{+} \mu^{-}$are extracted from data using a maximum likelihood fit of the dimuon invariant mass. To improve the sensitivity, the fit is performed simultaneously in three intervals of continuum BDT output designed to have equal signal efficiency of $18 \%$. These regions have different levels of background thus allowing to constrain its properties in the simultaneous fit. The likelihood includes various systematic uncertainties as nuisance parameters, but statistical uncertainties largely dominate. Total expected number of signal events according to the SM prediction is 41 and 5 for $N_{s}$ and $N_{d}$, respectively, equally distributed among the three intervals.

The fit projections are shown in Fig. 3. Without applying any boundary on the values of the fitted yields, the values determined by the fit are $N_{s}=16 \pm 12, N_{d}=-11 \pm 9$. The primary result of the analysis is obtained by applying the natural boundary of non-negative yields, for which the fit returns the values $N_{s}=11, N_{d}=0$.

The branching fraction $\mathcal{B}\left(B_{s}^{0} \rightarrow \mu^{+} \mu^{-}\right)$corresponding to the fitted yield with the constraint of non-negative values is $\left(0.9_{-0.8}^{+1.1}\right) \times 10^{-9}$ where the uncertainties are quoted for $68.3 \%$ confidence interval. The observed significance of the $B_{s}^{0} \rightarrow \mu^{+} \mu^{-}$signal is determined using pseudo-experiments and found to be 1.4 standard deviations while the corresponding expected significance for the SM prediction is 3.1 standard deviations. Pseudo-experiments are also used to evaluate the compatibility of the simultaneous fit result with the SM expectations. This results in a $p$ value of $0.048 \pm 0.002$, corresponding to 2.0 standard deviations.

Figure 4 shows the contours in the plane of $\mathcal{B}\left(B_{s}^{0} \rightarrow \mu^{+} \mu^{-}\right)$and $\mathcal{B}\left(B^{0} \rightarrow \mu^{+} \mu^{-}\right)$drawn for values of $-2 \Delta \ln (L)$ equal to $2.3,6.2$ and 11.8 , relative to the maximum of the likelihood $L$, allowing negative values of the branching fractions. The maximum within the physical boundary is shown with error bars indicating the $68.3 \%$ interval for the value of $\mathcal{B}\left(B_{s}^{0} \rightarrow \mu^{+} \mu^{-}\right)$. Also shown are the corresponding contours obtained in the combination of the results of the CMS and LHCb experiments [17], and the prediction based on the SM.

An upper limit is placed on the $B_{s}^{0} \rightarrow \mu^{+} \mu^{-}$branching fraction using the $\mathrm{CL}_{\mathrm{s}}$ method [19] at the 95\% confidence level: $\mathcal{B}\left(B_{s}^{0} \rightarrow \mu^{+} \mu^{-}\right)<3.0 \times 10^{-9}$. The limit is obtained under the hypothesis of background only, with $\mathcal{B}\left(B^{0} \rightarrow \mu^{+} \mu^{-}\right)$left as a free parameter in the fit. The expected limit is $1.8_{-0.4}^{+0.7} \times 10^{-9}$. 

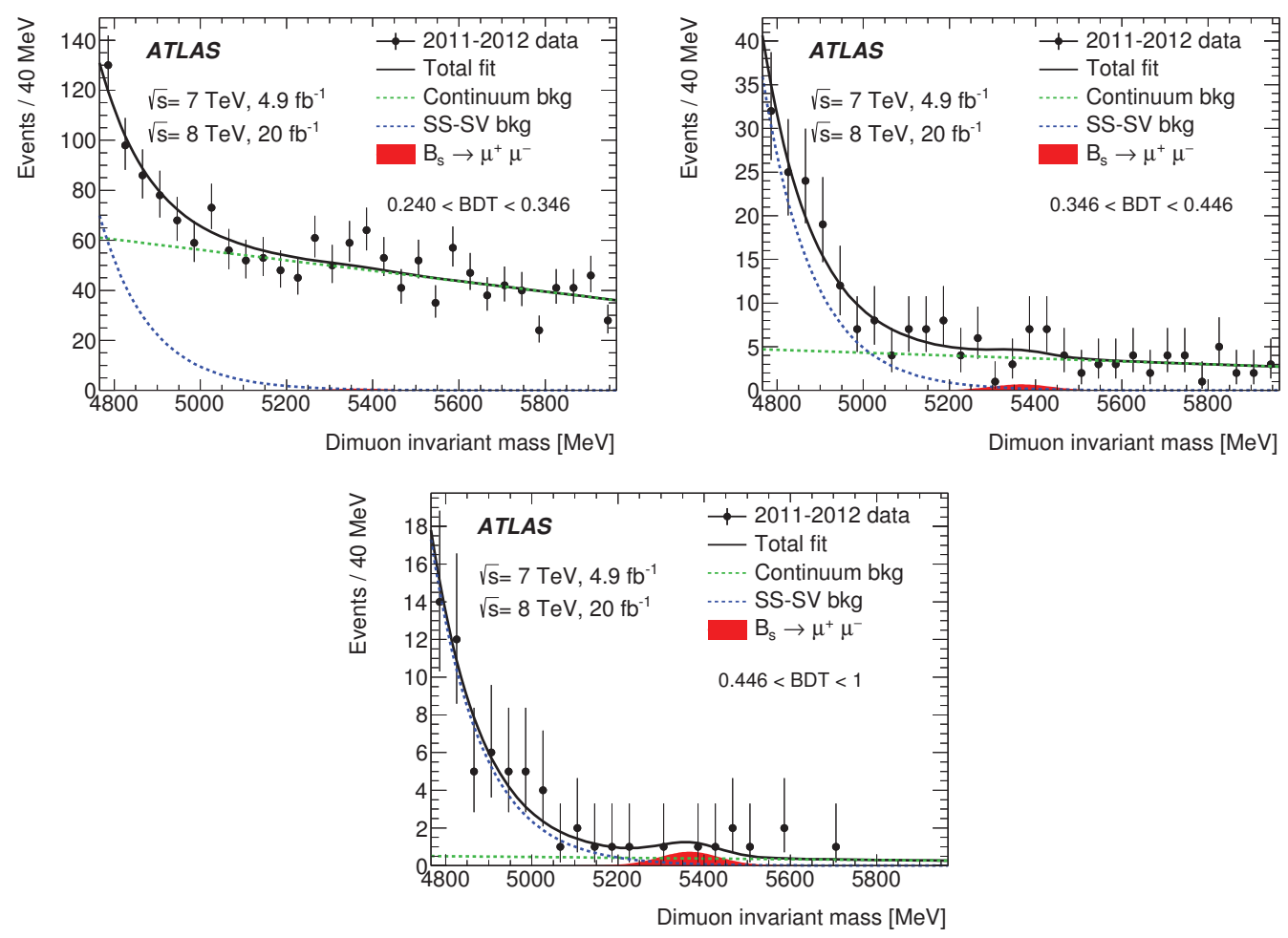

Figure 3. Dimuon invariant mass distributions in the unblinded data, in the three intervals of continuum-BDT output. Superimposed is the result of the maximum-likelihood fit, obtained imposing the boundary of nonnegative signal contributions. The total fit is shown as a black continuous line, the filled area corresponds to the observed signal component, the blue and green dashed lines to background components. Figure taken from [3].

An upper limit is also set on the branching fraction of the $B^{0}$ decay: $\mathcal{B}\left(B^{0} \rightarrow \mu^{+} \mu^{-}\right)<4.2 \times$ $10^{-10}(95 \% \mathrm{CL})$. This upper limit is above the SM prediction and also covers the central value of the combination of the measurements by CMS and LHCb [17]. Tension in the value of this branching fraction is reduced with the most recent result of $\mathrm{LHCb}$ [20] not included in the combination, where the upper limit $\mathcal{B}\left(B^{0} \rightarrow \mu^{+} \mu^{-}\right)<3.4 \times 10^{-10}(95 \% \mathrm{CL})$ is set.

\section{Conclusion}

Two analyses of $b$ hadron decays sensitive to new physics performed by ATLAS experiment are presented. The results of the angular analysis of $B^{0} \rightarrow \mu^{+} \mu^{-} K^{* 0}$ decay are found to agree with the SM predictions within three standard deviations across the whole studied range of $q^{2}$. In the rare $B_{(s)}^{0} \rightarrow \mu^{+} \mu^{-}$decays study, although the expected sensitivity of ATLAS measurement is comparable to that of LHCb and CMS, the observed signals are lower than those in the other experiments. This result is compatible with the SM expectations at level of two standard deviations. 


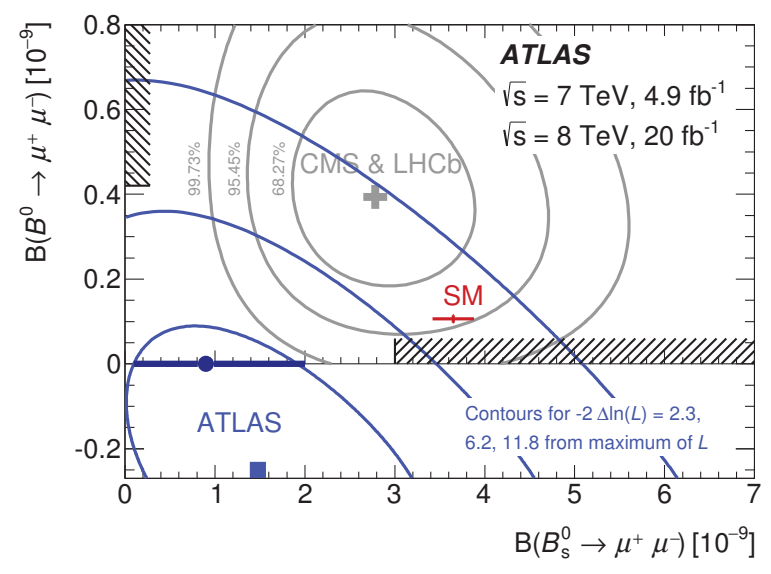

Figure 4. Contours in the plane $\mathcal{B}\left(B_{s}^{0} \rightarrow \mu^{+} \mu^{-}\right), \mathcal{B}\left(B^{0} \rightarrow \mu^{+} \mu^{-}\right)$for intervals of $-2 \Delta \ln (L)$ equal to $2.3,6.2$ and 11.8 relative to the absolute maximum of the likelihood, without imposing the constraint of non-negative branching fractions. Also shown are the corresponding contours for the combined result of the CMS and LHCb experiments, the SM prediction, and the maximum of the likelihood within the boundary of non-negative branching fractions, with the error bars covering the $68.3 \%$ confidence range for $\mathcal{B}\left(B_{s}^{0} \rightarrow \mu^{+} \mu^{-}\right)$. Figure taken from [3].

\section{References}

[1] ATLAS Collaboration, JINST 3, S08003 (2008)

[2] ATLAS Collaboration, ATLAS-CONF-2017-023, http://cds. cern.ch/record/2258146

[3] ATLAS Collaboration, Eur. Phys. J. C 76, 513 (2016)

[4] C. Patrignani et al. (Particle Data Group), Chin. Phys. C 40, 100001 (2016)

[5] LHCb Collaboration, Phys. Rev. Lett. 111, 191801 (2013)

[6] M. Ciuchini, M. Fedele, E. Franco, S. Mishima, A. Paul, L. Silvestrini, M. Valli, JHEP 06, 116 (2016)

[7] S. Descotes-Genon, L. Hofer, J. Matias, J. Virto, JHEP 12, 125 (2014)

[8] S. Jäger, J. Martin Camalich, JHEP 05, 043 (2013)

[9] LHCb Collaboration, JHEP 02, 104 (2016)

[10] CMS Collaboration, Phys. Lett. B 753, 424 (2016)

[11] J.T. Wei et al. (Belle Collaboration), Phys. Rev. Lett. 103, 171801 (2009)

[12] S. Wehle et al. (Belle Collaboration), Phys. Rev. Lett. 118, 111801 (2017)

[13] J.P. Lees et al. (BaBar Collaboration), Phys. Rev. D 93, 052015 (2016)

[14] C. Bobeth, M. Gorbahn, T. Hermann, M. Misiak, E. Stamou, M. Steinhauser, Phys. Rev. Lett. 112, 101801 (2014)

[15] CMS Collaboration, Phys. Rev. Lett. 111, 101804 (2013)

[16] LHCb Collaboration, Phys. Rev. Lett. 111, 101805 (2013)

[17] LHCb and CMS Collaborations, Nature 522, 68 (2015)

[18] A. Hocker et al., PoS ACAT, 040 (2007)

[19] A.L. Read, J. Phys. G 28, 2693 (2002)

[20] LHCb Collaboration, Phys. Rev. Lett. 118, 191801 (2017) 